\title{
Accurate Determination of the Limiting Anisotropy of Rhodamine 101. Implications for Its Use as a Fluorescence Polarization Standard
}

\author{
Telmo J. V. Prazeres, Alexander Fedorov, Sandrina P. Barbosa, José M. G. Martinho,* and \\ Mário N. Berberan-Santos* \\ Centro de Química-Física Molecular, Instituto Superior Técnico, Universidade Técnica de Lisboa, \\ 1049-001 Lisboa, Portugal
}

Received: November 5, 2007; Revised Manuscript Received: March 7, 2008

\begin{abstract}
The $\mathrm{S}_{1}-\mathrm{S}_{0}$ limiting anisotropy of a widely used fluorophore, rhodamine 101 , is determined with unprecedented accuracy. From time-resolved and steady-state fluorescence measurements in several solvents, it is shown that the limiting anisotropy of rhodamine 101 is for all practical purposes equal to the theoretical one-photon fundamental anisotropy value of $2 / 5$, both in rigid and in fluid media. This fact, along with the favorable chemical and photophysical properties of rhodamine 101, point to its use as a standard for fluorescence polarization measurements. It is also shown that if the excitation pulse can be considered a delta impulse with respect to the time scale of the anisotropy decay (but not necessarily to the time scale of the intensity decay), then no deconvolution procedure is needed for anisotropy decay analysis.
\end{abstract}

\section{Introduction}

Rhodamine 101 (also known as rhodamine 640), Chart 1, and derivatives retaining the same fluorophore are widely used as fluorescent probes. Rhodamine 101 was designed so that no rotation of the amino groups is possible, ${ }^{1,2}$ in the hope of achieving unit fluorescence quantum yield, but this was not fulfilled. ${ }^{3}$ Indeed, its fluorescence quantum yield, although high, is similar to that of rhodamine $6 \mathrm{G}^{3-5}$ and is in fact distinctly lower than $1.0,{ }^{4-6}$ which is the value initially reported. ${ }^{1,2,7}$ In room temperature acidified ethanol, where the dye is present in the cationic form, it has a fluorescence quantum yield of about $0.9^{6}$ and a single exponential fluorescence decay with a lifetime of 4.3 ns. $^{6}$ The cation and the zwitterion share a common fluorophore, whereas the lactone form has quite different photophysical properties. ${ }^{8}$

A precise determination of the $\mathrm{S}_{1}-\mathrm{S}_{0}$ limiting anisotropy (i.e., the highest measured $S_{1}-S_{0}$ anisotropy for a given molecule) of several xanthene dyes, including the rhodamines B, 6G and 101 , and fluorescein, $r_{0}=0.373 \pm 0.002$, was reported by Johansson, ${ }^{9}$ on the basis of both steady-state and time-resolved measurements. This begs the question as to why the limiting anisotropy is lower than the fundamental anisotropy, whose onephoton theoretical value is 0.4 for parallel absorption and emission transition moments.

The fact that the limiting anisotropy seldom attains the theoretical value of $2 / 5$ has been subject of considerable attention over the years; see, e.g., the classic work of Feofilov ${ }^{10}$ for an early view, and Valeur's book ${ }^{11}$ for a recent discussion. Possible reasons for the discrepancy fall in four categories: (i) instrumental effects (effect of wide angle collection and/or of polarizer misalignment, ${ }^{12,13}$ etc.); (ii) matrix-dependent effects (depolarization by light scattering, depolarization by stress-induced optical activity in solid glasses, depolarization by residual rotational motion); (iii) intermolecular effects (depolarization by radiative and/or nonradiative energy migration); (iv) intramolecular effects (mixed polarization bands, significantly

* Corresponding authors. E-mail: J.M.G.M., jgmartinho@ist.utl.pt; M.N.B., berberan@ist.utl.pt.
CHART 1: Possible Forms of Rhodamine 101<smiles>O=C1OC2(c3ccccc31)c1cc3c4c(c1Oc1c2cc2c5c1CCCN5CCC2)CCCN4CCC3</smiles>

Lactone

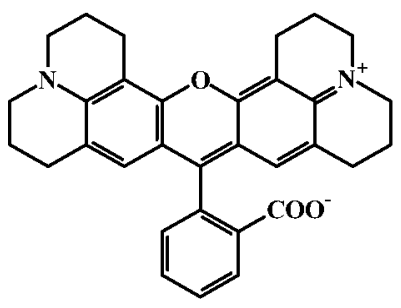

Zwitterion

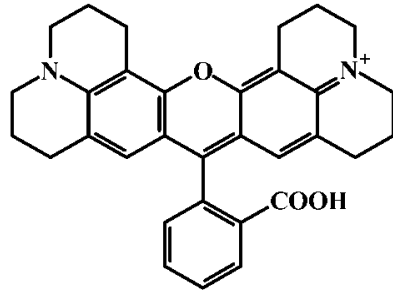

Cation different geometries for the Franck-Condon and emissive states implying noncoincident absorption and emission transition moments). It should be noted that the angle between the absorption and emission transition moments is related to the fundamental anisotropy by $\alpha=\arccos \left[\left(5 r_{0}+1\right) / 3\right]^{1 / 2}$ (that for $r_{0}$ close to $2 / 5$ reduces to $\left.\alpha=\left[{ }^{5} / 3\left({ }^{2} / 5-r_{0}\right)\right]^{1 / 2}\right)$; hence an anisotropy equal to $0.373 \pm 0.002$ implies an angle of $12.3 \pm$ $0.5^{\circ}$, which is small but nevertheless still significant, whereas an anisotropy equal to $0.400 \pm 0.005$ (the values above 0.4 having no physical meaning) implies an upper limit of $5^{\circ}$ for the angle, which for most purposes is essentially zero. In this way, and in the absence of significant systematic errors, a precision of 0.005 suffices for establishing if the angle between the absorption and emission transition moments is or is not essentially zero. The quest for a reliable value of the limiting anisotropy is therefore essentially the search of conditions where systematic errors are negligible at the required level of precision. 
In the case of the xanthene dyes, and after excluding the socalled librational motion as the cause for the deviations observed, the possibility of structural changes, admittedly not hindered by the matrix, was put forward as the explanation. ${ }^{9,14}$ Nevertheless, in a previous (but not quoted in refs 9 and 14) study by Szalay et al. $^{15}$ of fluorescein in glycerol-water mixtures at 30 ${ }^{\circ} \mathrm{C}$, a much higher limiting anisotropy of $0.392 \pm 0.005$ was obtained. Jameson et al. ${ }^{16}$ reported more recently for rhodamine $\mathrm{B}$ in a PMMA matrix at room temperature a similar value (0.393), again only marginally lower than 0.4 . Furthermore, in one time-resolved study conducted in our laboratory with rhodamine 101 in an acidified ethanol-water mixture, ${ }^{17}$ we also obtained a limiting anisotropy much closer to 0.4 than those previously published. ${ }^{9}$ On the other hand, early ${ }^{18,19}$ and more recent $^{20-22}$ time-resolved determinations of the limiting anisotropies of several xanthene dyes, including rhodamine 101, yielded values in the range $0.37-0.40$ with a typical precision of 0.02 , insufficient to decide between the two alternatives. All these observations prompted us to study in more detail the fluorescence polarization of rhodamine 101, and to determine its limiting value with unprecedented accuracy, quantifying at the same time the effect of some factors that may explain the substantially lower values reported in the literature.

\section{Experimental Methods}

Materials. High purity rhodamine 101 was purchased from Radiant Dyes, and PMMA (poly(methyl methacrylate)) with $\mathrm{MW}=120000 \mathrm{~g} \mathrm{~mol}^{-1}$, from Aldrich. All solvents used were of spectroscopic grade. Thin solid films were prepared by coating a quartz substrate at room temperature with mixtures of rhodamine 101 and PMMA in THF and letting the solvent to evaporate completely. PMMA concentration was $9.1 \mathrm{wt} \%$, and the concentration of rhodamine 101 was either $1.8 \times 10^{-7}$ or $1.6 \times 10^{-6} \mathrm{M}$. To $c a .2 \mathrm{~mL}$ of the mixture was added $20 \mu \mathrm{L}$ of a diluted solution of $\mathrm{HCl}$ in THF (ca. 1.1 M), to recover the cationic form of the dye. The final concentration of $\mathrm{HCl}$ in the mixture was $c a .10 \mathrm{mM}$. The dye concentration in the PMMA films was either $2.4 \times 10^{-6}$ or $2.2 \times 10^{-5} \mathrm{M}$. The final thickness of the films was of the order of a few micrometers $(1-20 \mu \mathrm{m})$.

Spectroscopic Measurements. Electronic absorption spectra were recorded on a Shimadzu Model 3101 UV-vis-NIR spectrometer and emission spectra on a SLM-Aminco 8100 Series 2 fluorometer. A standard $1 \mathrm{~cm}$ path length square section quartz cell was used for the measurements with the liquid solvents in a right angle geometry. A homemade support with goniometer was used for the thin film studies. For steady-state anisotropy measurements, typical instrumental bandwidths were $8 \mathrm{~nm}$ in both excitation and emission. All solutions used had an absorbance of 0.1 or lower for the steady-state measurements. Time-resolved picosecond fluorescence measurements were performed using the single-photon counting timing method with laser excitation. The setup consisted of a mode-locked Coherent Innova 400-10 argon ion laser that synchronously pumped a cavity dumped 710-2 dye (rhodamine 6G) laser, delivering 3-4 ps pulses at a repetition rate of $1.9 \mathrm{MHz}$. Intensity decay measurements were made by an alternate collection of impulse and decay, with the emission polarizer set at the magic angle position. Impulse was recorded slightly away from the excitation wavelength with a scattering suspension. For the decays a cutoff filter was used, effectively removing all excitation light. Anisotropy decay measurements were made by successive collection of the impulse and of the polarized components, with the emission polarizer set at the appropriate positions and with identical accumulation times for the two polarized components.

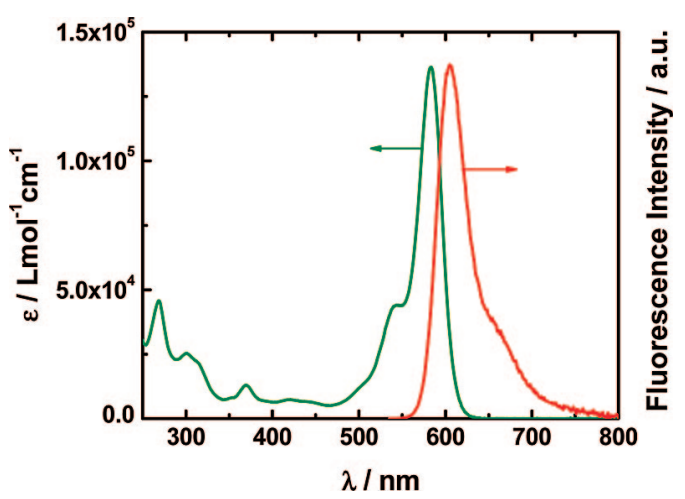

Figure 1. Absorption and corrected fluorescence $\left(\lambda_{\mathrm{exc}}=525 \mathrm{~nm}\right)$ spectra of rhodamine 101 in acidified ethylene glycol. Concentration of rhodamine 101: $4.1 \times 10^{-6} \mathrm{M}$ (absorption), $2.0 \times 10^{-6} \mathrm{M}$ (fluorescence).

The emission signal was first passed through a depolarizer, and then sent to a Jobin-Yvon HR320 monochromator with a grating of 100 lines/nm, and was recorded on a Hamamatsu 2809U-01 microchannel plate photomultiplier as a detector. With this setup, the $\mathrm{G}$ factor is equal to 1 . Time scales of 3.5-10 ps/channel were used. The instrument response function had an effective fwhm of 35 ps. Usually no fewer than 5000 counts were accumulated at the maximum channel. The numerical aperture for fluorescence collection was 0.18 (lens with a diameter of $18 \mathrm{~mm}$ and a focal length of $50 \mathrm{~mm}$ ); hence the half-angle $\theta$ was $10.4^{\circ}$ and paraxial conditions hold. The effect of a finite collection cone on the measured anisotropy was negligible at our precision level, as discussed in Appendix 1.

\section{Results and Discussion}

The fluorescence polarization of rhodamine 101 was studied at room temperature $\left(23^{\circ} \mathrm{C}\right)$ in two protic solvents of different viscosity, ethylene glycol (ethane-1,2-diol) $(19 \mathrm{mPa} \mathrm{s})^{23}$ and glycerol (propane-1,2,3-triol) $(1135 \mathrm{mPa} \mathrm{s}),{ }^{24}$ and also in a PMMA rigid matrix. The absorption and fluorescence spectra of rhodamine 101 (cationic form) are shown in Figure 1.

The excitation polarization spectrum of rhodamine 101 in glycerol at room temperature is displayed in Figure 2. Seven $\mathrm{S}_{n}-\mathrm{S}_{0}$ bands with distinctly different polarizations are observed above $250 \mathrm{~nm}$.

The $\mathrm{S}_{1}-\mathrm{S}_{0}$ absorption band corresponds to the region between 480 and $650 \mathrm{~nm}$, approximately. The $\mathrm{S}_{1}-\mathrm{S}_{0}$ steady-state anisotropy is always somewhat lower than $0.4(0.384 \pm 0.006$ for a concentration $1.1 \times 10^{-8} \mathrm{M}$ ) and decreases significantly with an increase in concentration, Figure 3.

The anisotropy decrease with concentration in the low concentration range displayed can be entirely ascribed to radiative energy migration (also called reabsorption), as studied in detail in refs 25 and 26, where it is also shown that the anisotropy is in general emission-wavelength dependent. From time-resolved measurements more detailed information is obtained with respect to the limiting anisotropy value. The anisotropy decay for a dilute solution $\left(1.1 \times 10^{-7} \mathrm{M}\right)$ is shown in Figure 4.

It is immediately seen that the zero-time anisotropy is much closer to 0.4 than the steady-state value, owing to the existence of a slow anisotropy decay time. From a direct fit to the anisotropy curve, in the time range $1.5-12.5 \mathrm{~ns}$, for which deconvolution is unnecessary (see Appendix 2), one obtains a zero-time anisotropy of $0.400 \pm 0.002$ and a decay time of 120 $\pm 6 \mathrm{~ns}$. The fluorescence intensity decay has a lifetime of 3.87 

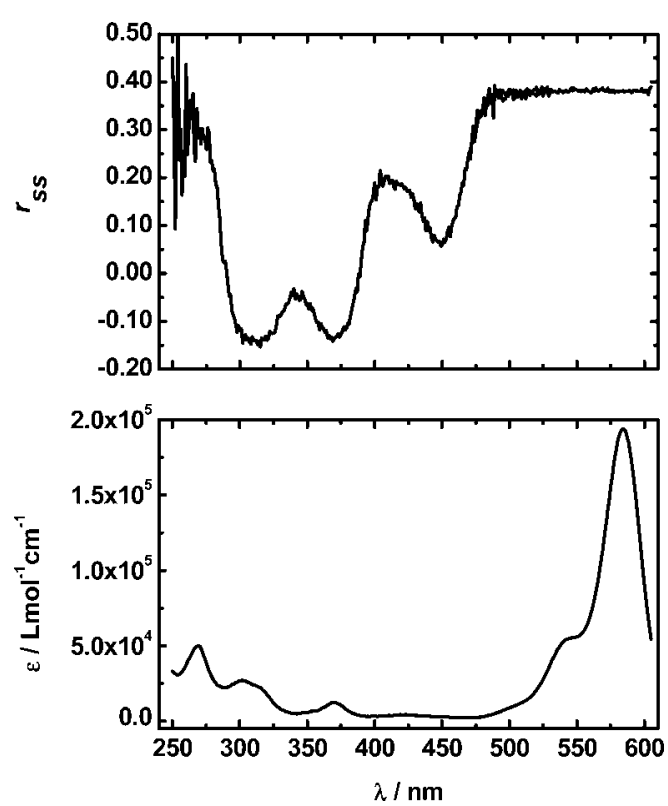

Figure 2. Anisotropy $\left(r_{\mathrm{ss}}\right)$ excitation spectrum $\left(\lambda_{\mathrm{em}}=615 \mathrm{~nm}\right)$ of rhodamine 101 in glycerol at $1.12 \times 10^{-7} \mathrm{M}$ (top) and the absorption spectrum of rhodamine 101 in glycerol at $7.61 \times 10^{-6} \mathrm{M}$ (bottom).

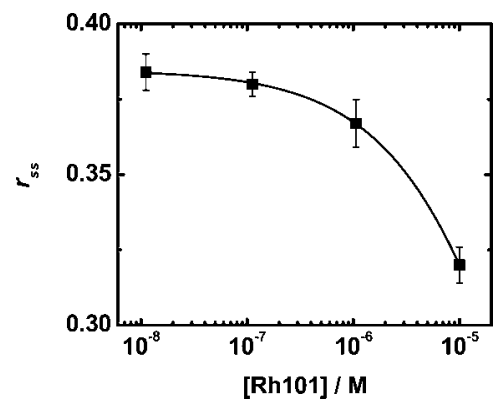

Figure 3. $\mathrm{S}_{1}-\mathrm{S}_{0}$ steady-state anisotropy $\left(r_{\mathrm{ss}}\right)$ in glycerol as a function of rhodamine 101 (Rh101) concentration. $\lambda_{\mathrm{exc}}=575 \mathrm{~nm}, \lambda_{\mathrm{em}}=615$ nm.

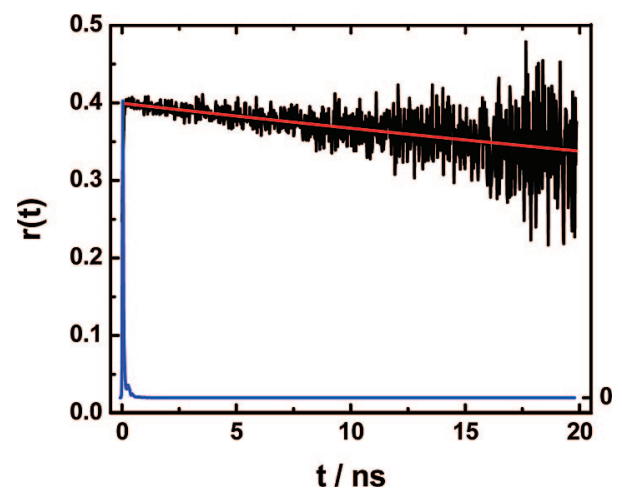

Figure 4. Experimental fluorescence anisotropy decay (black line), fitted anisotropy decay (red line), and instrument response function (blue line) for rhodamine 101 in glycerol $\left(1.1 \times 10^{-7} \mathrm{M}\right)$ at $23^{\circ} \mathrm{C}$. $\lambda_{\mathrm{exc}}=$ $575 \mathrm{~nm}$, and $\lambda_{\mathrm{em}}=615 \mathrm{~nm}$.

ns (with a small amplitude risetime of $0.41 \mathrm{ns,} \mathrm{owing} \mathrm{to} \mathrm{solvent}$ relaxation, as previously observed ${ }^{27}$ ). Calculation of the steadystate anisotropy from Perrin's equation, $r_{\mathrm{ss}}=r_{0} /(1+\tau / \theta)$, where $\theta$ is the anisotropy decay time, yields 0.388 , in good agreement with the observed value, $0.384 \pm 0.006$. The concentration dependence of the steady-state anisotropy, Figure 3, results from the decrease with concentration of the anisotropy decay time $\theta$ that reflects the extent of radiative energy migration. ${ }^{25,26}$
TABLE 1: Fluorescence lifetimes of Rhodamine 101 in Acidified Ethylene Glycol at $23{ }^{\circ} \mathrm{C}\left(\lambda_{\text {exc }}=575 \mathrm{~nm}, \lambda_{\mathrm{em}}=615\right.$ nm)

\begin{tabular}{ccccccc}
\hline concentration $\left(\mathrm{mol} \mathrm{dm}^{-3}\right)$ & $\tau_{1} / \mathrm{ns}$ & $\alpha_{1}$ & $\tau_{2} / \mathrm{ns}$ & $\alpha_{2}$ & $\alpha_{2} / \alpha_{1}$ & $\chi^{2}$ \\
\hline $1.0 \times 10^{-9}$ & 4.00 & 8.04 & 1.09 & -0.43 & -0.053 & 0.99 \\
$1.0 \times 10^{-8}$ & 4.01 & 6.06 & 0.91 & -0.34 & -0.056 & 0.99 \\
$1.0 \times 10^{-7}$ & 4.02 & 7.91 & 0.28 & -0.59 & -0.074 & 1.09 \\
$1.0 \times 10^{-6}$ & 4.07 & 8.04 & 0.40 & -0.70 & -0.087 & 1.18 \\
$0.97 \times 10^{-5}$ & 4.46 & 7.94 & 0.54 & -0.59 & -0.074 & 1.11 \\
$4.9 \times 10^{-5}$ & 4.98 & 7.77 & 0.75 & -0.24 & -0.031 & 1.04
\end{tabular}

Fluorescence intensity and anisotropy decays in acidified ethylene glycol, again at $23{ }^{\circ} \mathrm{C}$, were measured as a function of the solute concentration. The intensity decays were fitted with a sum of two exponentials, of which one had a negative amplitude, Table 1.

The variation of the two decay components with concentration is depicted in Figure 5.

The effect of radiative energy migration is clearly seen for concentrations as low as $10^{-6} \mathrm{M}$, with the lifetime with positive amplitude increasing significantly above $4.0 \mathrm{~ns}$. The negative component reflects both solvent relaxation and radiative energy migration. Both the lifetime increase and the negative amplitude component are expected on the basis of previous studies of radiative energy migration in rhodamine 101 solutions. , $^{45,26}$

The anisotropy decay of a $1.0 \times 10^{-9} \mathrm{M}$ solution is shown in Figure 6.

The anisotropy decay is well fitted by a single exponential. The fitted anisotropy decay times and zero-time anisotropies are plotted as a function of concentration in Figures 7 and 8, respectively.

The anisotropy decay time values are much lower than those observed in glycerol, as the much lower viscosity of ethylene glycol implies a significant depolarization by rotational motion. The low concentration value, $2.5 \mathrm{~ns}$, is similar to that measured for rhodamine $6 \mathrm{G}$ in the same solvent at room temperature. ${ }^{28}$ The effect of radiative energy migration is again clearly seen in Figure 7 for concentrations as low as $10^{-6} \mathrm{M}$. On the other hand, the limiting anisotropy values, Figure 8 , are concentrationindependent, and cluster around 0.4, with $r_{0}=0.400 \pm 0.004$.

As mentioned, in a previous time-resolved study conducted in our laboratory with rhodamine 101 in an acidified ethanol-water $(85: 15 \mathrm{v} / \mathrm{v})$ mixture at room temperature, ${ }^{17}$ a limiting anisotropy value of $0.392 \pm 0.007$ was obtained. The rotational correlation time was in this case 290 ps.

The steady-state anisotropy, as well as the fluorescence intensity and anisotropy decays of rhodamine 101 were also

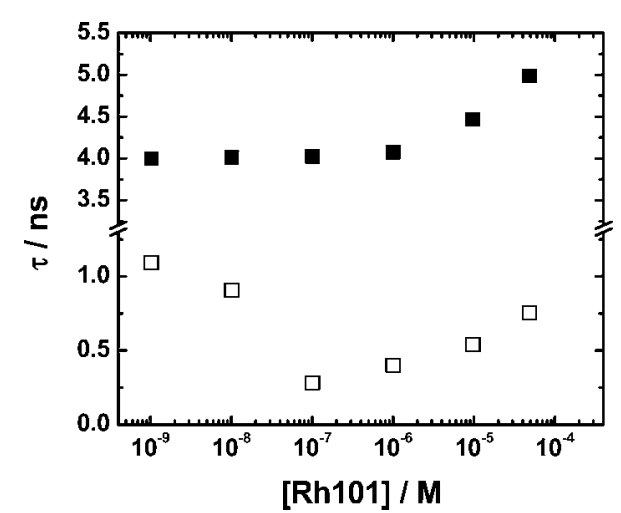

Figure 5. Lifetimes ( $\tau_{1}$, filled squares) and rise times ( $\tau_{2}$, empty squares) of rhodamine 101 (Rh 101) in acidified ethylene glycol as a function of concentration. 


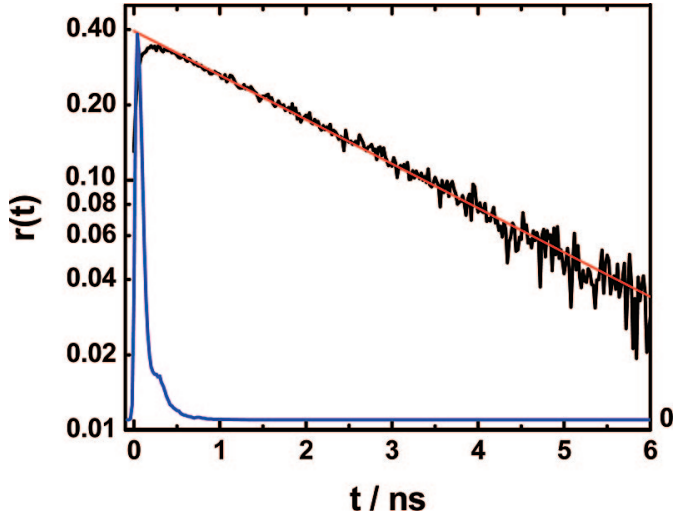

Figure 6. Experimental fluorescence anisotropy decay (black line), fitted anisotropy decay (red line), and instrument response function (blue line) for rhodamine 101 in ethylene glycol $\left(1.0 \times 10^{-9} \mathrm{M}\right)$ at $23{ }^{\circ} \mathrm{C}$. $\lambda_{\text {exc }}=575 \mathrm{~nm}, \lambda_{\mathrm{em}}=615 \mathrm{~nm}$.

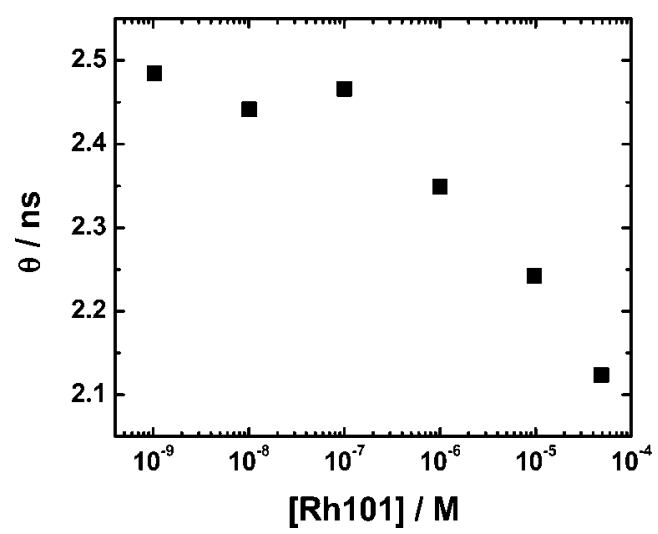

Figure 7. Anisotropy decay times of rhodamine 101 (Rh 101) in ethylene glycol at $23{ }^{\circ} \mathrm{C}$ as a function of concentration. $\lambda_{\text {exc }}=575$ $\mathrm{nm}, \lambda_{\mathrm{em}}=615 \mathrm{~nm}$.

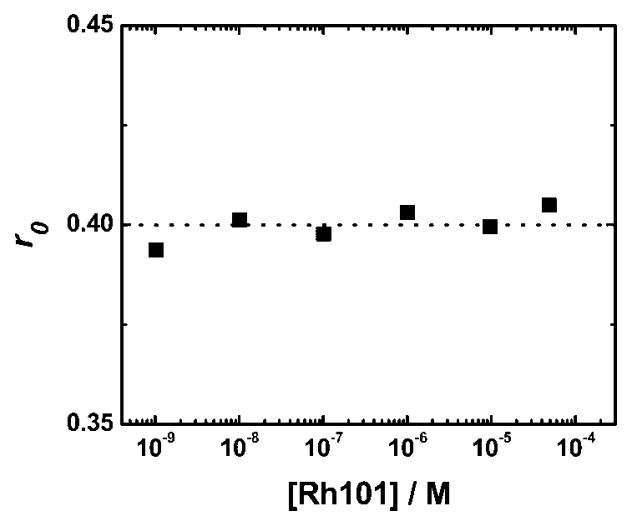

Figure 8. Zero-time anisotropies of rhodamine 101 (Rh 101) in ethylene glycol at $23^{\circ} \mathrm{C}$ as a function of concentration. $\lambda_{\text {exc }}=575$ $\mathrm{nm}, \lambda_{\mathrm{em}}=615 \mathrm{~nm}$.

measured in a PMMA thin film at room temperature, Figure 9. For better accuracy, three steady-state anisotropy excitation spectra were measured for the $S_{1}-S_{0}$ band: between 475 and $515 \mathrm{~nm}$ with $\lambda_{\mathrm{em}}=585 \mathrm{~nm}$, between 481 and $585 \mathrm{~nm}$ with $\lambda_{\mathrm{em}}$ $=615 \mathrm{~nm}$, and between 550 and $610 \mathrm{~nm}$ with $\lambda_{\mathrm{em}}=635 \mathrm{~nm}$. The corresponding anisotropies were essentially identical, 0.394 $\pm 0.005,0.404 \pm 0.004$, and $0.396 \pm 0.008$, respectively. The intensity decay was close to a single exponential, with a lifetime of 3.8 ns. As can be seen in Figure 9, the anisotropy "decay" is perfectly horizontal for several tens of nanoseconds, with a constant value $r(t)=0.396 \pm 0.014(1.5-12.5 \mathrm{~ns}$ time range $)$.

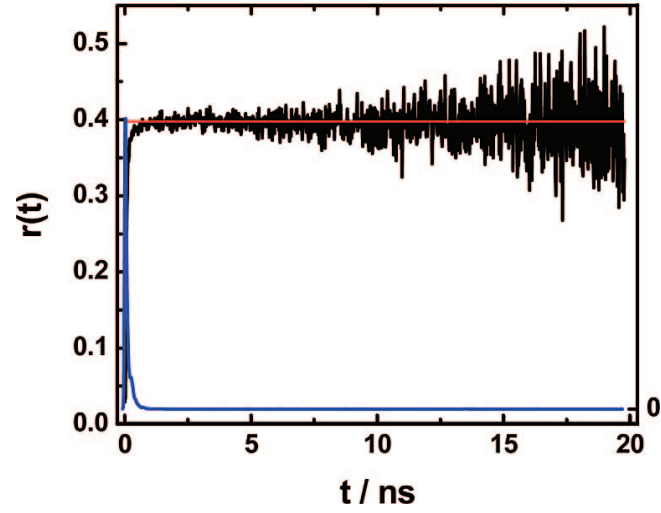

Figure 9. Experimental fluorescence anisotropy decay (black line), fitted anisotropy decay (red line), and instrument response function (blue line) for rhodamine 101 in a PMMA film $\left(2 \times 10^{-6} \mathrm{M}\right)$ at $23^{\circ} \mathrm{C}$. $\lambda_{\text {exc }}$ $=575 \mathrm{~nm}, \lambda_{\mathrm{em}}=615 \mathrm{~nm}$.

From the described set of measurements, it is concluded that the limiting anisotropy of rhodamine 101 is for all purposes equal to the theoretical one-photon fundamental anisotropy value of $2 / 5$, both in rigid and in fluid media. This fact, along with the favorable chemical and photophysical properties of rhodamine 101 , point to its use as a standard for fluorescence polarization measurements. Indeed, only a handful of polarization standards have been proposed, ${ }^{29,30}$ and none displays the theoretical upper limit.

\section{Conclusions}

The $S_{1}-S_{0}$ limiting anisotropy of rhodamine 101 was determined with high accuracy. From time-resolved and steadystate fluorescence measurements in ethanol, ethylene glycol, glycerol, and PMMA, it is shown that the limiting anisotropy of rhodamine 101 is for all purposes equal to the theoretical one-photon fundamental anisotropy value of $2 / 5$, both in rigid and in fluid media. It is therefore concluded that there are no significant structural differences between the ground and the emissive states of rhodamine 101. Our study demonstrates the effect of concentration and viscosity on steady-state and timeresolved anisotropy measurements, and how these parameters need to be controlled to obtain accurate limiting anisotropy values. The fact that the limiting anisotropy of rhodamine 101 is $2 / 5$, along with the favorable chemical and photophysical properties of rhodamine 101, point to its use as a standard for fluorescence polarization measurements.

It was also shown that if the excitation pulse can be considered a delta impulse with respect to the time scale of the anisotropy decay (but not necessarily to the time scale of the intensity decay), then no deconvolution procedure is needed to fit the anisotropy decay, whose analysis becomes very simple.

Acknowledgment. This work was supported by Fundação para a Ciência e a Tecnologia (FCT, Portugal) and POCI 2010 (FEDER) within project POCI/QUI/58515/2004 and through the Associated Laboratory IN - Instituto de Nanociências e Nanotecnologias. T.J.V.P. was supported by a grant from project POCI/QUI/58515/2004.

\section{Appendix 1}

When excitation of an isotropic sample is made with vertically polarized light, and the absorption and emission transition dipoles coincide, the fluorescence anisotropy only attains the theoretical maximum value of $2 / 5$ for directions perpendicular 
to the oscillating electric field, i.e., the horizontal directions. For directions deviating from the horizontal by an angle $\varphi$, the anisotropy is given by

$$
r=\frac{2 \cos ^{2} \varphi}{3+2 \cos ^{2} \varphi}
$$

For a given numerical aperture, the collection lens subtends a cone with half angle $\theta$. Owing to refraction, and for small angle (paraxial conditions) the half angle of the emission cone inside the cell is $\theta / n$, where $n$ is the refractive index of the sample's medium, hence $\varphi \leq \theta / n$. The average anisotropy corresponding to an integration over the cone's solid angle is approximately given by

$$
r=\frac{2}{5}\left[1-\frac{1}{5}\left(\frac{\theta}{n}\right)^{2}\right]
$$

where $\theta$ is expressed in radians. In this way, for $\theta=10.4^{\circ}$, and using $n=1.47$ (glycerol), the average anisotropy takes the value 0.399 , which is for our purposes indistinguishable from $2 / 5$.

\section{Appendix 2}

For an excitation pulse of finite duration, $P(t)$, the fluorescence anisotropy is given by ${ }^{31,32}$

$$
r(t)=\frac{P \otimes\left(r_{\delta} f\right)}{P \otimes f}
$$

where $r_{\delta}(t)$ is the anisotropy decay in response to a delta pulse, and $f(t)$ is the fluorescence intensity decay in response to the same pulse, and $\otimes$ stands for the convolution between two functions,

$$
P \otimes f=\int_{0}^{t} P(u) f(t-u) \mathrm{d} u
$$

The excitation pulse is essentially zero for times larger than a given instant $t_{0}$ (in our case $80 \mathrm{ps}$ ) hence eq A2.1 becomes, for $t>t_{0}$

$$
r(t)=\frac{\int_{0}^{t_{0}} P(u) r_{\delta}(t-u) f(t-u) \mathrm{d} u}{\int_{0}^{t_{0}} P(u) f(t-u) \mathrm{d} u}
$$

If both the anisotropy and the fluorescence intensity decay exponentially,

$$
\begin{gathered}
r_{\delta}(t)=r_{0} \exp (-t / \theta) \\
f(t)=\exp (-t / \tau)
\end{gathered}
$$

then eq A2.3 becomes

$$
r(t)=\alpha r_{\delta}(t)
$$

where $\alpha$ is a constant,

$$
\alpha=\frac{\int_{0}^{t_{0}} P(u) \exp \left[\left(\frac{1}{\tau}+\frac{1}{\theta}\right) u\right] \mathrm{d} u}{\int_{0}^{t_{0}} P(u) \exp \left(\frac{u}{\tau}\right) \mathrm{d} u}
$$

The anisotropy decay time $\theta$ can thus be simply obtained from the "raw" anisotropy for $t>t_{0}$, using eq A2.6, rewritten as

$$
\ln r(t)=\ln \left(\alpha r_{0}\right)-\frac{t}{\theta}
$$

If $\theta \gg \tau$ or if $\min (\theta, \tau) \gg t_{0}$, then $\alpha=1$, and $r_{0}$ is also immediately obtained from $r(t)$ for $t>t_{0}$; see, e.g., Figures 6 and 9, without the need to perform any of the common deconvolution procedures. ${ }^{33}$ If none of these inequalities apply, $\alpha$ can be estimated from eq A2.7 once $\theta$ and $\tau$ are determined, and therefore $r_{0}$ can in principle be still evaluated from eq A2.8. It is interesting to note that eq A2.6 can be written as

$$
r(t)=r\left(t_{0}\right) \exp \left(-\frac{t-t_{0}}{\theta}\right) \quad\left(t>t_{0}\right)
$$

i.e., if $t_{0}$ is viewed as the time origin, then the initial anisotropy is $r\left(t_{0}\right)$.

The result $r(t)=r_{\delta}(t)\left(t>t_{0}\right)$ is still valid for more complex decays, provided $r_{\delta}(t)$ does not change significantly for a period with duration $\Delta t=t_{0}$, i.e., if the pulse can be considered a delta impulse with respect to the time scale of the anisotropy decay (but not necessarily to the time scale of the intensity decay). When this is not valid, one must resort to eq A2.1. For the extreme case of very fast anisotropy decays, this implies performing the full convolution analysis within the excitation pulse, as was successfully done in ref 34 .

\section{References and Notes}

(1) Drexhage, K. H. Laser Focus 1973, 9, 35

(2) Drexhage, K. H. J. Res. Natl. Bur. Stand. 1976, 80A, 421.

(3) Magde, D.; Rojas, G. E.; Seybold, P. G. Photochem. Photobiol. 1999, $70,737$.

(4) Kubin, R. F.; Fletcher, A. N. J. Lumin. 1982, 27, 455.

(5) Arden, J.; Deltau, G.; Huth, V.; Kringel, U.; Peros, D.; Drexhage, K. H. J. Lumin. 1991, 48 \& 49, 352.

(6) Nunes Pereira, E. J.; Berberan-Santos M. N.; Vincent, M.; Gallay, J.; Fedorov, A.; Martinho, J. M. G. J. Chem. Phys. 1999, 110, 1600.

(7) Karstens, T.; Kobs, K. j. Phys. Chem. 1980, 84, 1871.

(8) Karpiuk, J.; Grabowski, Z. R.; De Schryver, F. C. J. Phys. Chem. 1994, $98,3247$.

(9) Johansson, L. B.-Å. J. Chem. Soc. Faraday Trans. 1990, 86, 2103.

(10) Feofilov, P. P. The Physical Basis of Polarized Emission; Consultants Bureau: New York, 1961, p. 110.

(11) Valeur, B. Molecular Fluorescence. Principles and Applications; Wiley-VCH: Weinheim, 2002, p. 137.

(12) Weber, G. J. Opt. Soc. Am. 1956, 46, 962.

(13) Tschanz, H. P.; Binkert, Th. J. Phys. E 1976, 9, 1131.

(14) Ryderfors, L.; Mukhtar, E.; Johansson, L. B.-Å. J. Fluoresc. 2007, $17,466$.

(15) Szalay, L.; Gáti, L.; Sárkány, B. Acta Phys. Acad. Sci. Hung. 1962, 14, 217. cited in Albrecht, A. C. Prog. React. Kinet. 1970, 5, 301.

(16) Jameson, D. M.; Croney, J. C.; Moens, P. D. J. Meth. Enzymol. 2003, 360,1 .

(17) Barbosa, S. P. Ph. D. Thesis; IST: Lisbon, 2005.

(18) Fleming, G. R.; Morris, J. M.; Robinson, G. W. Chem. Phys. 1976, 17,91 .

(19) Bauer, R. K.; Balter, A. J. Lumin. 1979, 20, 249.

(20) Megens, M.; Sprik, R.; Wegdam, G. H.; Lagendik, A. J. Chem. Phys. 1997, 107, 493.

(21) Lettinga, M. P.; Zuilhof, H.; van Zandvoort, M. A. M. J. Phys. Chem. Chem. Phys. 2000, 2, 3697.

(22) Prazeres, T. J. V.; Fedorov, A.; Martinho, J. M. G. J. Phys. Chem. B 2004, 108, 9032.

(23) Sun, T.; Teja, A. S. J. Chem. Eng. Data 2003, 48, 198.

(24) Cook, R. L.; King, H. E.; Herbst, C. A.; Herschbach, D. R. J. Chem. Phys. 1994, 100, 5178.

(25) Nunes Pereira, E. J.; Berberan-Santos, M. N.; Martinho, J. M. G. J. Chem. Phys. 1996, 104, 8950.

(26) Berberan-Santos, M. N.; Nunes Pereira, E. J.; Martinho, J. M. G., In Resonance Energy Transfer; Andrews D. L., Demidov, A. A., Eds.; Wiley: Chichester, 1999.

(27) Visser, A. J. W. G.; Vos, K.; van Hoek, A.; Santgema, J. S. J. Phys. Chem. 1988, 92, 759.

(28) Siegel, J.; Suhling, K.; Lévêque-Fort, S.; Webb, S. E. D.; Davis, D. M.; Phillips, D.; Sabharwal, Y.; French, P. M. W. Rev. Sci. Instrum. 2003, 74, 182 .

(29) Thompson, R. B.; Gryczynski, I.; Malicka, J. BioTechniques 2002, 32,34 . 
(30) DeRose, P.; Smith, M. V.; Mielenz, K.; Blackburn, D. H.; Kramer, G. W. J. Lumin. 2008, 128, 257.

(31) Berberan-Santos, M. N. J. Lumin. 1991, 50, 83.

(32) Valeur, B.; Berberan-Santos, M. N.; Martin, M. M., In Analytical Methods in Supramolecular Chemistry.: Schalley A. Ed.; Wiley-VCH: Weinheim, 2007
(33) O'Connor, D. V.; Phillips, D. Time-Correlated Single Photon Counting; Academic Press: Orlando, 1984, pp. 252-283.

(34) Berberan-Santos, M. N.; Choppinet, P.; Fedorov, A.; Jullien, L.; Valeur, B. J. Am. Chem. Soc. 2000, 122, 11876.

JP710625J 